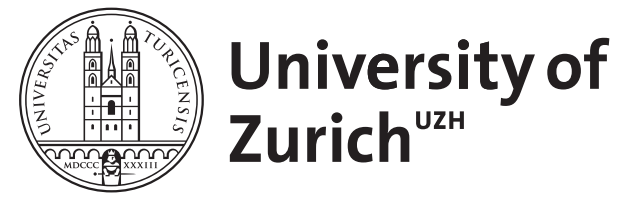

Zurich Open Repository and Archive

University of Zurich

University Library

Strickhofstrasse 39

CH-8057 Zurich

www.zora.uzh.ch

Year: 2012

\title{
Traumatic brain injury and disturbed sleep and wakefulness
}

\author{
Baumann, C R
}

DOI: https://doi.org/10.1007/s12017-012-8178-x

Posted at the Zurich Open Repository and Archive, University of Zurich

ZORA URL: https://doi.org/10.5167/uzh-67166

Journal Article

Published Version

Originally published at:

Baumann, C R (2012). Traumatic brain injury and disturbed sleep and wakefulness. NeuroMolecular Medicine, 14(3):205-212.

DOI: https://doi.org/10.1007/s12017-012-8178-x 


\title{
Traumatic Brain Injury and Disturbed Sleep and Wakefulness
}

\author{
Christian R. Baumann
}

Received: 29 September 2011/ Accepted: 3 March 2012/Published online: 23 March 2012

(C) Springer Science+Business Media, LLC 2012

\begin{abstract}
Traumatic brain injury is a frequent condition worldwide, and sleep-wake disturbances often complicate the course after the injuring event. Current evidence suggests that the most common sleep-wake disturbances following traumatic brain injury include excessive daytime sleepiness and posttraumatic hypersomnia, that is, increased sleep need per $24 \mathrm{~h}$. The neuromolecular basis of posttraumatic sleep pressure enhancement is not entirely clear. First neuropathological and clinical studies suggest that impaired hypocretin (orexin) signalling might contribute to sleepiness, but direct or indirect traumatic injury also to other sleep-wake modulating systems in the brainstem and the mesencephalon is likely. Posttraumatic insomnia may be less common than posttraumatic sleepiness, but studies on its frequency revealed conflicting results. Furthermore, insomnia is often associated with psychiatric comorbidities, and some patients with posttraumatic disruption of their circadian rhythm may be misdiagnosed as insomnia patients. The pathophysiology of posttraumatic circadian sleep disorders remains elusive; however, there is some evidence that reduced evening melatonin production due to traumatic brain damage may cause disruption of circadian regulation of sleep and wakefulness.
\end{abstract}

Keywords Sleep - Vigilance - Traumatic brain injury . Hypocretin · Orexin · Melatonin

C. R. Baumann $(\bowtie)$

Department of Neurology, University Hospital Zurich,

Frauenklinikstrasse 26, 8091 Zurich, Switzerland

e-mail: christian.baumann@usz.ch
Traumatic Brain Injury: Definition, Aetiology, and Symptoms

Traumatic brain injury (TBI) is one of the major causes of death and disability throughout the world, particularly among children and young adults. In the literature, the annual incidence rate of department visits for TBI has been reported to be around 400/100,000 (Langlois et al. 2006). An estimated number of 1.6 million people sustain TBI each year in the United States, accounting for 52,000 deaths and 80,000 patients suffering from irreversible neurological impairment (Sosin et al. 1996; Bruns and Hauser 2003). Data from Europe indicate an annual incidence of hospitalized and fatal TBI of 235 per 100,000 (Tagliaferri et al. 2006).

TBI is caused by an outside force traumatically injuring the brain. The Demographics and Clinical Assessment Working Group of the International and Interagency Initiative toward Common Data Elements for Research on Traumatic Brain recently introduced the following definition of TBI (Menon et al. 2010):

TBI is defined as an alteration in brain function, or other evidence of brain pathology, caused by an external force.

Thereby, alteration in brain function is defined as one of the followings: (1) any period of loss of or a decreased level of consciousness, (2) any loss of memory for events immediately before (retrograde amnesia) or after the injury, (3) neurological deficits (such as weakness, paresis or plegia, loss of balance, change in vision, dyspraxia, sensory loss, aphasia), or (4) any alteration in mental state at the time of the injury (such as confusion, disorientation, slowed thinking) [1]. Furthermore, other evidence of brain pathology may include visual, neuroradiological, or laboratory confirmation 
of damage to the brain. Lastly, external forces may include one of the followings: (1) the head being struck by an object or striking an object, (2) the brain undergoing an acceleration-deceleration movement without direct external trauma to the head, (3) a foreign body penetrating the brain, (4) forces generated from events such as a blast or explosion, or (5) other force yet to be defined [1].

Thus, non-traumatic brain injuries such as stroke, tumours, and inflammation, which do not involve external mechanical force, must be clearly distinguished from TBI. Common causes of TBI include vehicle and sport accidents, falls, and any form of violence. The classification of TBI is based either on the mechanism (closed versus penetrating TBI), on the severity (as assessed by scales such as the Glasgow Coma Scale, or by neuroimaging of the brain), or on the localization of the trauma (e.g. localized in a specific brain area versus widespread).

In general, there are two types of traumatic brain damage (Maas et al. 2008). Primary damage relates to external forces, as a consequence of rapid acceleration or deceleration, direct impact, penetrating objects, or blast waves. Primary damage includes shearing injuries of white-matter tracts leading to diffuse axonal injury, focal contusions, haematomas, and oedema. On a cellular level, primary damage contains membrane and ion channel dysfunction as well as conformational protein changes. Each type of traumatic brain injury can initiate a variety of pathophysiological mechanisms, leading to secondary brain damage. These secondary cascades include free radical generation, gene activation, neurotransmitter release, calcium-mediated damage, mitochondrial dysfunction, and inflammatory responses. Combinations of these mechanisms may lead to cellular damage not only in the region of direct impact, but also in neighbouring and more distant brain areas.

In the acute stage of TBI, loss of consciousness or amnesia or both are the hallmark symptoms. The diagnosis of TBI (as opposed to head injury) is established on the basis of these clinical symptoms. Other symptoms are dependent on the type, severity, and localization of TBI. Typical symptoms of all forms of TBI include headache, nausea and vomiting, disturbed motor coordination, dizziness, cognitive disturbances, mood changes, blurred vision, tinnitus, fatigue, and changes in sleep patterns. With increasing TBI severity, symptoms become more severe and may sustain for longer times. Depending on the localization of trauma and of mass lesions such as intracranial haemorrhage, other symptoms such as anisocoria, coma, epileptic seizures, abnormal posturing, and focal neurological deficits can occur. An important contributor to fatal outcome after TBI is the increase in intracranial pressure. Signs of elevated intracranial pressure include sopor and coma, paralysis of limbs, dilated pupils, and autonomic failure with bradycardia, increased arterial blood pressure, and respiratory depression. These symptoms develop over hours to days after trauma, which necessitates treatment and monitoring in an intensive care unit.

Improvement of neurological function usually occurs for two or more years after the trauma but is fastest during the first 6 months. Most patients with TBI have milder injuries, but residual deficits in these patients are not infrequent either (Thornhill et al. 2000). Residual symptoms after TBI include impaired consciousness, hyposmia and other focal neurological deficits, posttraumatic epilepsy and movement disorders, hormonal disturbances, cognitive deficits, psychiatric disorders, and sleep-wake disturbances (SWD).

On the other hand, SWD may negatively affect neurological sequelae in TBI patients. For instance, it has been shown that sustained attention is impaired in TBI patients with SWD (Bloomfield et al. 2010). In TBI with underlying obstructive sleep apnoea, sustained attention and memory are both worse than in TBI patients without sleepdisordered breathing (Wilde et al. 2007). In the same line, a study in 443 patients with mild TBI revealed that patients with SWD are more likely to suffer from concomitant headaches, depressive symptoms, and irritability (Chaput et al. 2009).

Posttraumatic SWD have been widely neglected by the scientific literature for many years. Accordingly, medical textbooks and Internet encyclopaedias provide a long list of complications and sequelae after TBI, but many of them still fail to mention sleep-wake problems after traumatic brain injury. In the next part, this chapter will give a short overview on posttraumatic SWD, particularly posttraumatic sleepiness.

\section{Sleep-Wake Disturbances are Common After Traumatic Brain Injury}

TBIs in humans are heterogeneous with reference to type, localization, and severity of trauma, which makes it difficult to systematically study posttraumatic SWD. Most of the few published studies on posttraumatic SWD were retrospective and in selected populations with some degree of inclusion bias and therefore gave no insights into the frequency of SWD following TBI. The first systematic and prospective studies on posttraumatic SWD have been published in the last few years. In 65 consecutive patients and 6 months after TBI, we systematically recorded SWD by means of interviews, questionnaires, and electrophysiological sleep laboratory examinations (Baumann et al. 2007). In 47 patients (72 \% of the population), traumarelated SWD have been identified, which were not present prior to the trauma. SWD occurred irrespective of the localization or the severity of the trauma. The most 
prevalent sleep-wake disturbance following TBI was impaired daytime vigilance (excessive daytime sleepiness and fatigue, in $55 \%$ ). Similarly, increased sleep need per $24 \mathrm{~h}$ ( $\geq 2 \mathrm{~h}$ more than before TBI) was also common (observed in $22 \%$ ). On the other hand, insomnia was found only in $5 \%$ of the TBI patients.

Another prospective clinical and sleep laboratory study in 87 patients at least 3 months (64 \pm 118 months) after TBI found SWD in $46 \%$ of the examined population (Castriotta et al. 2007). The authors applied nocturnal polysomnography, multiple sleep latency tests, the psychomotor vigilance test, and validated scales such as the Epworth sleepiness scale. Excessive daytime sleepiness was the most common finding (25\%). In this study, it was defined as mean sleep latencies on multiple sleep latency test (MSLT) below $10 \mathrm{~min}$. Psychomotor vigilance test results were worse in patients with low mean sleep latencies. In detail, $23 \%$ of patients suffered from obstructive sleep apnoea, $6 \%$ with narcolepsy (typical cataplexy was not reported), and $7 \%$ with periodic limb movements in sleep.

Data on the long-term outcome of sleep-wake disturbances after TBI, however, are even sparser. To better understand the natural history of posttraumatic sleepiness and other sleep-wake disturbances after TBI, we extended the observation period of our prospective study from 6 months to 3 years (Kempf et al. 2010). Fifty-one of the 65 TBI patients of the original study were included and examined by means of interviews and questionnaires including the Epworth sleepiness scale and the Fatigue severity scale. Compared to 6 months after trauma, the prevalence of (subjective) posttraumatic sleepiness decreased markedly from 28 to $12 \%$. On the other hand, we identified more patients suffering from fatigue: $35 \%$ of TBI patients were diagnosed with fatigue as compared to $17 \% 6$ months after trauma. Therefore, at least in TBI, there might be a continuum between fatigue and sleepiness: in the course after TBI, sleep propensity during daytime might fade, giving way to a rather unspecific tiredness that may become more prominent.

\section{Disturbed Night-Time Sleep After Traumatic Brain Injury}

After the wars in Iraq and in Afghanistan, disruption of nocturnal sleep and its behavioural sequelae became of interest for both researchers and politicians. However, there are limited data on insomnia in war veterans, in whom traumatic brain injury and posttraumatic stress disorder often coexist (Wallace et al., 2011). Contrariwise, most disparate epidemiological findings have been published on posttraumatic insomnia, which is often associated with anxiety or depression or both. A prospective study based on sleep questionnaires found a high prevalence (30\%) of insomnia and poor sleep quality in 50 patients after TBI (Fichtenberg et al. 2002). In a retrospective study comprising a heterogeneous population of 184 somnolent subjects suffering from TBI or head-neck trauma (whiplash injury), $45 \%$ of the patients reported insomnia ('disturbed nocturnal sleep'), mostly due to nocturnal pain (Guilleminault et al. 2000). In a questionnaire study, more than $50 \%$ of 452 TBI patients reported insomnia symptoms (Ouellet et al. 2006). In another study by the same group, using subjective and objective measures of sleep quality, 14 TBI patients were compared to 14 healthy good sleepers (Ouellet et al., 2006b). The authors found that TBI patients with insomnia have a tendency to overestimate their sleep disturbance compared to polysomnographic measures of sleep, indicating that insomnia symptoms may be overestimated after TBI. The same was suggested by Gosselin and colleagues (2009), showing that polysomnographic recordings in concussed athletes could not confirm subjective complaints about poor nocturnal sleep quality. In the same line, our prospective study that included actigraphy and polysomnography studies 6 months after trauma revealed insomnia in only $5 \%$ of 65 consecutive patients (Baumann et al. 2007). Insomnia has been shown to persist 3 years after TBI (Kempf et al. 2010), and insomnia symptoms are associated with concomitant headache, depressive symptoms, and irritability (Chaput et al. 2009).

\section{Disturbed Daytime Vigilance After Traumatic Brain Injury}

The terms 'hypersomnia' and 'excessive daytime sleepiness' are often used synonymously for increased sleep propensity, even in official classifications. However, in a strict sense, hypersomnia is defined as increased sleep need per $24 \mathrm{~h}$, whereas excessive daytime sleepiness refers to increased daytime sleep propensity, that is, difficult to fight sleep during daytime. This misunderstanding of the term hypersomnia might be one reason why studies on posttraumatic hypersomnia are sparse. In our own prospective study in 65 TBI patients, we defined posttraumatic hypersomnia as increased sleep need $\geq 2 \mathrm{~h}$ compared to pre-TBI conditions (Baumann et al. 2007). Six months after trauma, we found hypersomnia in $22 \%$ of our patients, and most patients with posttraumatic sleep-wake disturbances suffered either from hypersomnia or from excessive daytime sleepiness/fatigue (Baumann et al. 2007). TBI patients on a job with strict working schedules and TBI patients with young children more often suffered from excessive daytime sleepiness, whereas students, adults without a job, and elderly patients more often suffered from hypersomnia. Furthermore, an association between severity of TBI and the presence of hypersomnia was found. In the light of 
these findings, it could be hypothesized whether the primary sleep-wake disorder after TBI may be hypersomnia. Thus, patients whose psychosocial environment allows extended sleeping times per $24 \mathrm{~h}$ may avoid increased sleep propensity at daytime, whereas in patients who cannot compensate for the increased sleep need, excessive daytime sleepiness may occur.

In contrast to hypersomnia, posttraumatic sleepiness has been examined in several studies. However, there are limitations also in this regard. The assessment of excessive daytime sleepiness is hampered by methodological issues despite the presence of the so-called objective tests. Excessive daytime sleepiness can be assessed with questionnaires such as the Epworth sleepiness scale (subjective excessive daytime sleepiness), and with sleep laboratory tests such as the multiple sleep latency test (objective excessive daytime sleepiness). It has been shown that there is no statistically or clinically significant association between Epworth scores and mean sleep latency on the multiple sleep latency test (Benbadis et al. 1999). One possible explanation is that the subjective Epworth sleepiness scale and the objective multiple sleep latency test may evaluate different, complementary aspects of sleepiness. Unfortunately, studies on heterogeneous samples of TBI patients used different subjective and objective measures, which make comparisons between populations impossible. In our own population, we found that many of our $65 \mathrm{TBI}$ patients underestimate their sleep propensity, making objective tests indispensable. Unfortunately, there is still no consensus which diagnostic test should be used for the measurement of excessive daytime sleepiness. Many authors doubt the accuracy of the multiple sleep latency test to diagnose and quantify excessive daytime sleepiness, whereas questionnaires are limited by their subjective character (Johns et al., 2000).

In 1983, based on a polygraphic study, Guilleminault and colleagues alluded to the importance of recognizing excessive daytime sleepiness as a residual symptom after TBI, and they found an association of posttraumatic sleepiness with sleep apnoea in a significant portion of their patients $(\mathrm{Gu}-$ illeminault et al. 1983). Others measured excessive daytime sleepiness with the multiple sleep latency test and found posttraumatic sleepiness in almost $50 \%$ (33 of 71) of their patients with different types of brain injury (traumatic, ischaemic, haemorrhagic, anoxic) (Masel et al. 2001). In a small study using polysomnography and MSLT in 10 patients with TBI and excessive daytime sleepiness, underlying sleep-wake disorders such as sleep apnoea and periodic limb movement syndrome were found in all tested patients (Castriotta et al., 2001). Our own prospective data, however, suggested that in most TBI patients, the majority of cases with posttraumatic sleepiness cannot be explained by underlying sleep-wake, neurological, or other disorders (Baumann et al. 2007). Alternatively, these data suggest that posttraumatic excessive daytime sleepiness is directly related to the neuronal injury itself. Subjective posttraumatic sleepiness (as assessed with the Epworth sleepiness scale) was found in $28 \%$ of 65 consecutive TBI patients and objective posttraumatic sleepiness (as assessed by the multiple sleep latency test) in $25 \%$. Subjective and/or objective posttraumatic sleepiness was found in $38 \%$, and the concurrence of both subjective and objective posttraumatic sleepiness was found in only 9 patients. Similarly, there was no significant correlation between mean sleep latency on MSLT and Epworth sleepiness scale. Another recent prospective clinical and sleep laboratory study in 87 patients at least 3 months after trauma, however, confirmed the high frequency of posttraumatic sleepiness (Castriotta et al. 2007). The authors found sleep-wake disturbances in $46 \%$ of the examined population, with posttraumatic sleepiness as the most common finding ( $25 \%)$.

\section{Disrupted Circadian Rhythm After Traumatic Brain Injury}

The findings pertaining to circadian sleep-wake disorders following TBI are sparse and rather inconclusive. Anecdotal reports on a posttraumatic delayed sleep phase syndrome have been published, but a study of 10 patients using questionnaires, sleep diaries, polysomnography, and saliva melatonin measurements failed to provide evidence of any shift in circadian timing of sleep subsequent to TBI (Quinto et al. 2000; Steele et al. 2005). Patients with this circadian rhythm disorder usually generally fall asleep some hours after midnight and have difficulty waking up in the morning. Contrariwise, a recent study in 42 minor TBI patients with complaints of insomnia has been examined by actigraphy, saliva melatonin, and oral temperature measurements (Ayalon et al. 2007). The authors found circadian sleep-wake disorders in $36 \%$ of these patients. Two types of circadian sleep-wake disorders were observed: delayed sleep phase syndrome and irregular sleep-wake pattern, that is, frequent changes of bedtimes over weeks and months. The observation of frequent circadian sleep-wake disorders following TBI further supports the assumption that posttraumatic insomnia might be overestimated.

\section{Pathophysiology of Posttraumatic Sleep-Wake Disturbances-Mostly Unknown}

Aetiology and pathophysiology of disrupted sleep and wakefulness following traumatic brain injury are mostly unknown, but are most likely multifactorial. Damage to the brain in human TBI involves a huge variety of localizations, and neurological and psychiatric sequelae that might 
contribute to SWD are equally disperse. Excessive daytime sleepiness, which belongs to the most common posttraumatic SWD, may be a good example to illustrate thisrecent neuromolecular studies have begun to elucidate the multifactorial pathophysiology of excessive daytime sleepiness.

Excessive daytime sleepiness following traumatic brain injury may be related to physical disability and increased exhaustion, but also to psychiatric symptoms such as depression and anxiety. Also, epilepsy and its treatments, as well as treatment for chronic pain can contribute to posttraumatic excessive daytime sleepiness.

Last but not least, it is unknown whether injury to specific brain regions might be responsible for the evolution of posttraumatic sleepiness. For instance, it is conceivable that lesions to wake-promoting brain areas might contribute to sleepiness after TBI. Descriptions of injury in TBI often focus on the midbrain and subthalamus, but hypothalamic and brainstem injuries are also common: one early study showed damage to the hypothalamus in $42 \%$ of deceased TBI patients, and brainstem regions are also commonly involved (Crompton et al., 1971; Crompton et al., 1971b). Similarly, there is evidence of pituitary and hypothalamic dysfunction after TBI (Karavitaki et al. 2006; Schneider et al. 2007). The exact pathophysiology of traumatic damage to these midbrain structures is unknown. Both types of traumatic brain damage_as introduced above-might contribute (Maas et al. 2008). These mechanisms may lead to cellular damage not only in the region of direct impact, but also in distant brain areas such as the midbrain.

In the acute period after TBI, low and undetectable cerebrospinal fluid levels of the wake-promoting neurotransmitter hypocretin (orexin) have been observed (Baumann et al. 2005). Hypocretins are produced by a small and distinct population of neurons in the posterior hypothalamus.
Fig. 1 Photomicrographs depicting the relationship between gliosis (GFAP staining of astrocytes) and hypocretin neurons. a Diaminobenzidine staining of a typical astrocyte in a control brain. Astrocyte density is much higher in the lateral hypothalamus of a TBI brain $\left(\mathbf{b}_{1}\right)$ than in the same region of a control patient $\left(\mathbf{c}_{1}\right)$. Double immunofluorescence staining shows that along with the reduction in the number of hypocretin neurons (green), there is an increase in red GFAP-labelled astrocytes (arrow) and fibres in TBI $\left(\mathbf{b}_{2}\right)$ compared to control subjects $\left(\mathbf{c}_{2}\right)$. Bar $30 \mu \mathrm{m}$ (Color figure online)
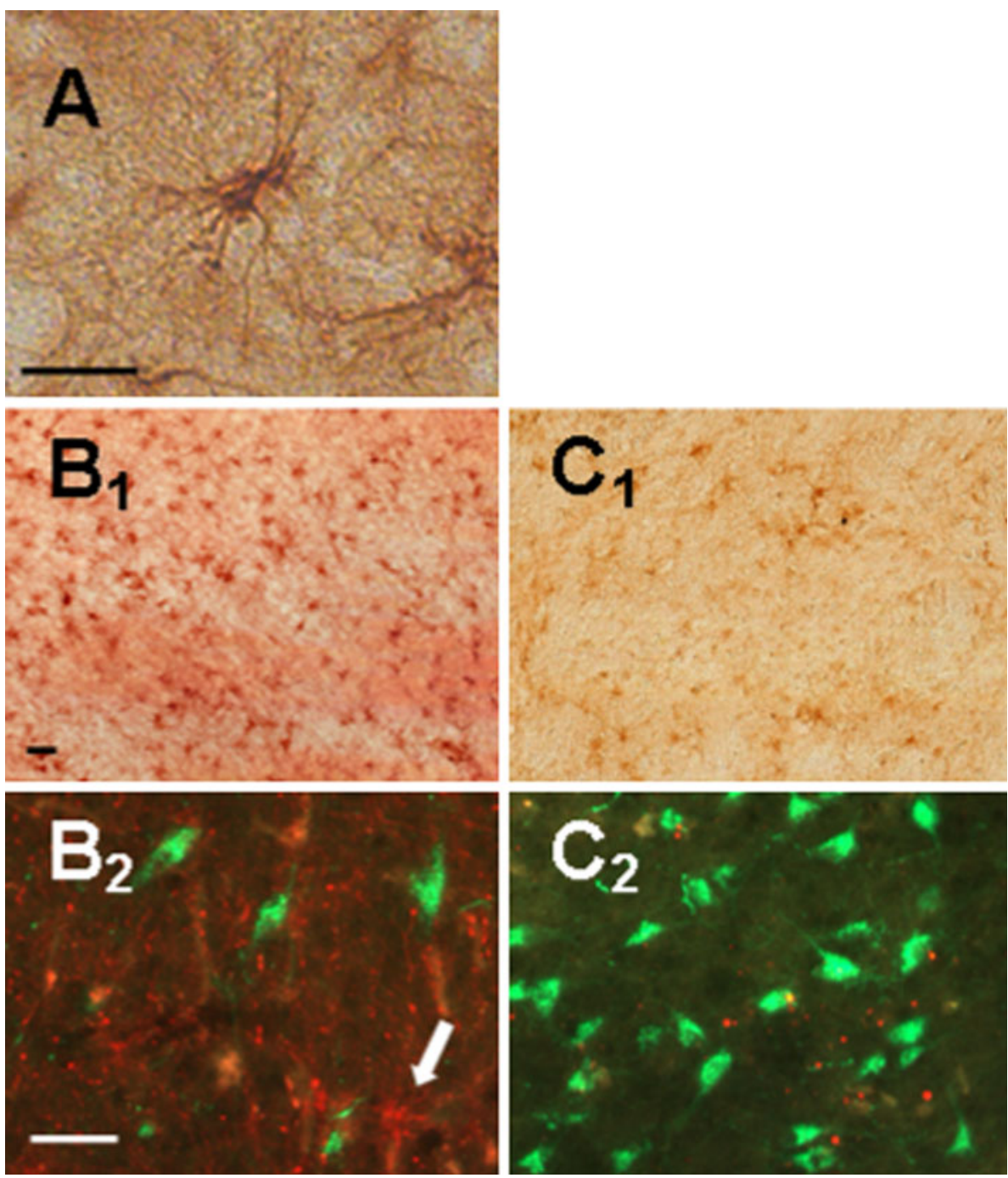
Fig. 2 Caudal-rostral distributions of hypocretin neurons in all TBI patients and controls. Each graph displays the total number of hypocretin neurons ( $y$ axis) per section (x axis). Caudal sections are displayed on the left side, rostral sections on the right side
Control 1 Left Side
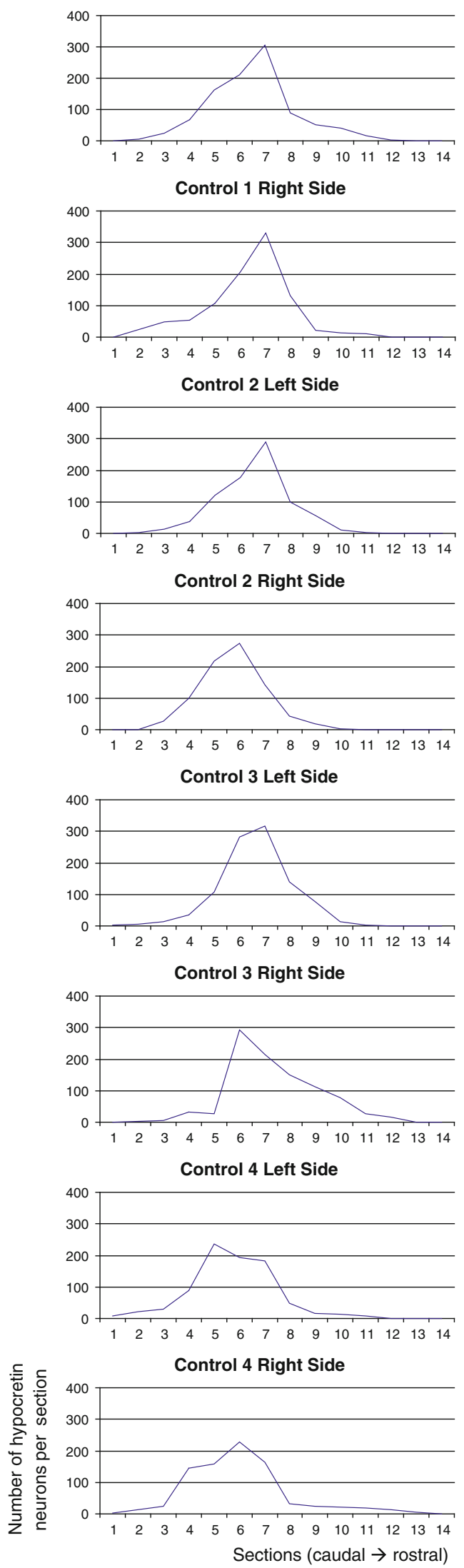

Patient 1 Left Side
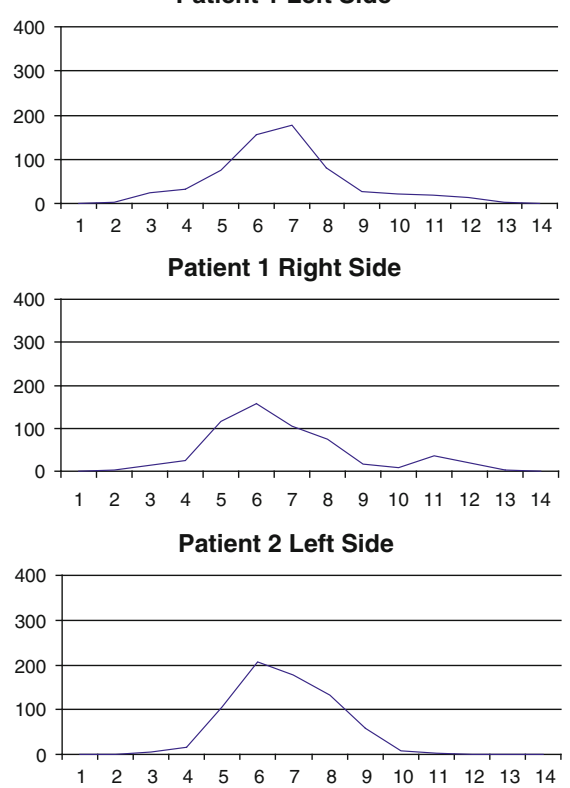

Patient 2 Right Side

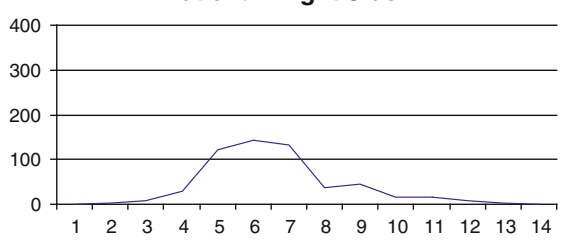

Patient 3 Left Side
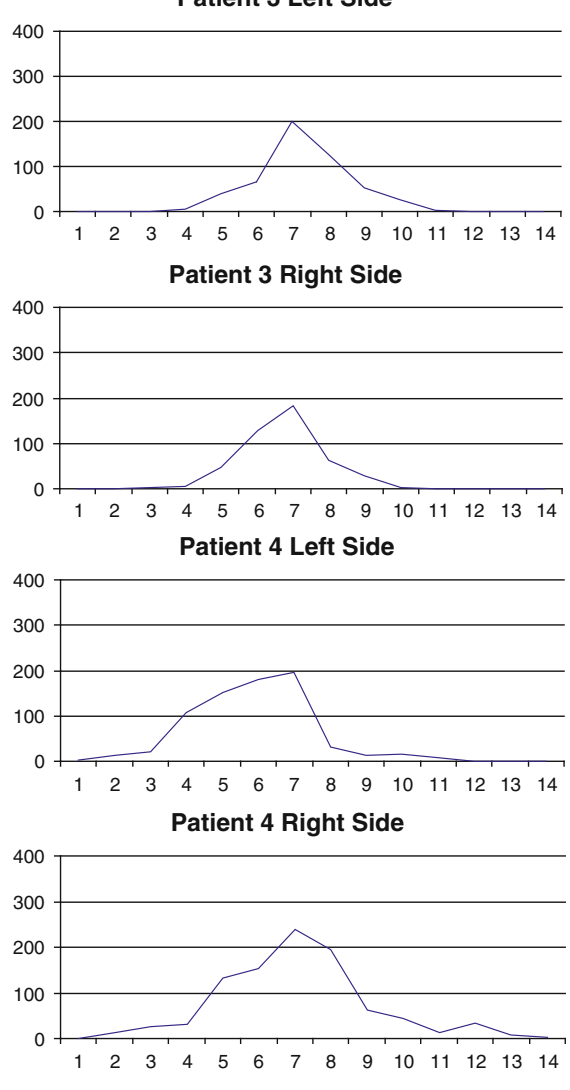
Six months after TBI, hypocretin levels recovered. In a subset of patients, however, this recovery was only partial (Baumann et al. 2007). Furthermore, in the same patients, there was an association between low hypocretin levels and posttraumatic sleepiness. Together with the early reports on hypothalamic damage in TBI, this finding led to the hypothesis of hypocretin neuronal loss in TBI. Our first preliminary study on brains of 4 deceased TBI patients ( $51 \pm 19$ years, death occurred 7-42 days after trauma) and of 4 controls ( $69 \pm 18$ years) confirmed this assumption and revealed a $27 \%$ loss of hypocretin neurons in deceased TBI patients (Fig. 1) (Baumann et al. 2009). Thus, although the number of examined patients was low, and although TBI patients were younger, they had significantly less hypocretin cells. Among the TBI patients, the number of hypocretin neurons differed moderately between the left and right sides of the hypothalamus and between individuals, reflecting the asymmetry and heterogeneity of TBI. However, throughout the hypothalamus, hypocretin neurons were distributed in a normal pattern, which suggests that traumatic hypothalamic damage is not restricted to specific areas, but involves the whole region (Fig. 2). Many hypocretin neurons in TBI patients were small and pyknotic, and the density of hypocretin fibres appeared reduced. Furthermore, we found intense perivascular hypocretin immunoreactivity in the hypothalami of TBI patients, possibly representing phagocytized hypocretin peptide following damage to the hypocretin neurons.

This injury, on the other hand, was not limited to the hypocretin neurons as there was a partial loss of neighbouring neurons producing melanin-concentrating hormone $(\mathrm{MCH})$ and gliosis throughout the hypothalamus of TBI patients (Fig. 1).

Still, it is unlikely that reduced hypocretin signalling is the only factor to cause sleepiness after TBI. About one-third of TBI patients have brainstem injuries, often near the midbrain tegmentum, that may disrupt ascending monoaminergic or cholinergic wake-promoting pathways (Crompton et al., 1971b). Loss of the MCH neurons may also contribute to the pathophysiology of posttraumatic sleep-wake disturbances as these cells are hypothesized to be involved in the regulation of REM sleep.

A recent study using transcranial magnetic stimulation found signs of cortical hypoexcitability in TBI with objective excessive daytime sleepiness (Nardone et al. 2011). In detail, they found higher resting motor thresholds and more pronounced short-latency intracortical inhibition in those patients than in control subjects, concluding that cortical hypoexcitability may reflect the deficiency of the excitatory hypocretin system.

Altogether, these findings strongly suggest that TBI can injure the hypothalamus. Although the clinical significance of a $27 \%$ loss of wake-promoting hypocretin neurons remains unclear, these findings further suggest thatprobably together with lesions in other wake-promoting neurotransmitter systems in the brainstem-reduced hypocretin signalling may contribute to the persistent sleepiness often seen in TBI patients.

The exact pathophysiology of posttraumatic circadian sleep-wake disorders remains elusive. However, a recent observational study comparing 23 patients with TBI to 23 matched healthy subjects assessed polysomnographic sleep measures, salivary dim light melatonin onset (DLMO) time, and self-reported sleep quality, anxiety, and depression (Shekleton et al. 2010). In this study, TBI patients showed significantly lower levels of evening melatonin production, together with more frequent complaints of sleep problems, and symptoms of depression or anxiety. The authors concluded that reduced evening melatonin production due to traumatic brain damage may indicate a disruption to circadian regulation of sleep and wakefulness.

\section{Conclusion}

In the field of sleep medicine, there is growing interest in posttraumatic sleep-wake disturbances, particularly posttraumatic sleepiness. However, there is still a lack of studies regarding the pathophysiology, epidemiology, clinical characteristics, and treatment of posttraumatic sleep-wake disturbances. In particular, the neuromolecular basis of posttraumatic sleep-wake disturbances will need a thorough work-up.

\section{References}

Ayalon, L., Borodkin, K., Dishon, L., Kanety, H., \& Dagan, Y. (2007). Circadian rhythm sleep disorders following mild traumatic brain injury. Neurology, 68, 1136-1140.

Baumann, C. R., Bassetti, C. L., Valko, P. O., Haybaeck, J., Keller, M., Clark, E., et al. (2009). Loss of hypocretin (orexin) neurons with traumatic brain injury. Annals of Neurology, 66, 555-559.

Baumann, C. R., Stocker, R., Imhof, H. G., et al. (2005). Hypocretin-1 (orexin A) deficiency in acute traumatic brain injury. Neurology, $65,147-149$.

Baumann, C. R., Werth, E., Stocker, R., Ludwig, S., \& Bassetti, C. L. (2007). Sleep-wake disturbances 6 months after traumatic brain injury: A prospective study. Brain, 130, 1873-1883.

Benbadis, S. R., Mascha, E., Perry, M. C., et al. (1999). Association between the Epworth sleepiness scale and the multiple sleep latency test in a clinical population. Annals of Internal Medicine, 130, 289-292.

Bloomfield, I. L., Espie, C. A., \& Evans, J. J. (2010). Do sleep difficulties exacerbate deficits in sustained attention following traumatic brain injury? Journal of the International Neuropsychological Society, 16, 17-25.

Bruns, J., Jr, \& Hauser, W. A. (2003). The epidemiology of traumatic brain injury: A review. Epilepsia, 44, 2-10. 
Castriotta, R. J., \& Lai, J. M. (2001). Sleep disorders associated with traumatic brain injury. Archives of Physical Medicine and Rehabilitation, 82, 1403-1406.

Castriotta, R. J., Wilde, M. C., Lai, J. M., Atanasov, S., Masel, B. E., \& Kuna, S. T. (2007). Prevalence and consequences of sleep disorders in traumatic brain injury. Journal of Clinical Sleep Medicine, 3, 349-356.

Chaput, G., Giguère, J. F., Chauny, J. M., et al. (2009). Relationship among subjective sleep complaints, headaches, and mood alterations following a mild traumatic brain injury. Sleep Medicine, 10, 713-716.

Crompton, M. R. (1971a). Brainstem lesions due to closed head injury. Lancet, 1, 669-673.

Crompton, M. R. (1971b). Hypothalamic lesions following closed head injury. Brain, 94, 165-172.

Fichtenberg, N. L., Zafonte, R. D., Putnam, S., et al. (2002). Insomnia in a post-acute brain injury sample. Brain Injury, 16, 197-206.

Gosselin, N., Lassonde, M., Petit, D., Leclerc, S., Mongrain, V., Collie, A., et al. (2009). Sleep following sport-related concussions. Sleep Medicine, 10, 35-46.

Guilleminault, C., Faull, K. F., Miles, L., \& van den Hoed, J. (1983). Posttraumatic excessive daytime sleepiness: A review of 20 patients. Neurology, 33, 1584-1589.

Guilleminault, C., Yuen, K. M., Gulevich, M. G., et al. (2000). Hypersomnia after head-neck trauma: A medicolegal dilemma. Neurology, 54, 653-659.

Johns, M. W. (2000). Sensitivity and specificity of the multiple sleep latency test (MSLT), the maintenance of wakefulness test and the epworth sleepiness scale: Failure of the MSLT as a gold standard. Journal of Sleep Research, 9, 5-11.

Karavitaki, N., Wass, J., Henderson Slater, J. D., \& Wade, D. (2006). A case of post-traumatic isolated ACTH deficiency with spontaneous recovery 9 months after the event. Journal of Neurology, Neurosurgery and Psychiatry, 77, 276-277.

Kempf, J., Werth, E., Kaiser, P. R., Bassetti, C. L., \& Baumann, C. R. (2010). Sleep-wake disturbances 3 years after traumatic brain injury. Journal of Neurology, Neurosurgery and Psychiatry, 81, $1402-1405$.

Langlois, J. A., Rutland-Brown, W., \& Thomas, K. E. (2006). Traumatic brain injury in the United States: Emergency department visits, hospitalizations, and deaths. Atlanta: Centers for Disease Control and Prevention, National Center for Injury Prevention and Control.

Maas, A. I., Stocchetti, N., \& Bullock, R. (2008). Moderate and severe traumatic brain injury in adults. Lancet Neurology, 7, $728-741$

Masel, B. E., Scheibel, R. S., Kimbark, T., \& Kuna, S. T. (2001). Excessive daytime sleepiness in adults with brain injuries. Archives of Physical Medicine and Rehabilitation, 82, $1526-1532$.
Menon, D. K., Schwab, K., Wright, D. W., \& Maas, A. I. (2010). Demographics and clinical assessment working group of the international and interagency initiative toward common data elements for research on traumatic brain injury and psychological health. Archives of Physical Medicine and Rehabilitation, 91, 1637-1640.

Nardone, R., Bergmann, J., Kunz, A., Caleri, F., Seidl, M., Tezzon, F., et al. (2011). Cortical excitability changes in patients with sleepwake disturbances after traumatic brain injury. Journal of Neurotrauma, 28, 1165-1171.

Ouellet, M. C., Beaulieu-Bonneau, S., \& Morin, C. M. (2006). Insomnia in patients with traumatic brain injury: Frequency, characteristics, and risk factors. The Journal of Head Trauma Rehabilitation, 21, 199-212.

Ouellet, M. C., \& Morin, C. M. (2006). Subjective and objective measures of insomnia in the context of traumatic brain injury: A preliminary study. Sleep Medicine, 7, 486-497.

Quinto, C., Gellido, C., Chokroverty, S., \& Masdeu, J. (2000). Posttraumatic delayed sleep phase syndrome. Neurology, 54, 250-252.

Schneider, H. J., Aimaretti, G., Kreitschmann-Andermahr, I., et al. (2007). Hypopituitarism. Lancet, 369, 1461-1470.

Shekleton, J. A., Parcell, D. L., Redman, J. R., Phipps-Nelson, J., Ponsford, J. L., \& Rajaratnam, S. M. (2010). Sleep disturbance and melatonin levels following traumatic brain injury. Neurology, 74, 1732-1738.

Sosin, D. M., Sniezek, J. E., \& Thurman, D. J. (1996). Incidence of mild and moderate brain injury in the United States, 1991. Brain Injury, 10, 47-54.

Steele, D. L., Rajaratnam, S. M., Redman, J. R., \& Ponsford, J. L. (2005). The effect of traumatic brain injury on the timing of sleep. Chronobiology International, 22, 89-105.

Tagliaferri, F., Compagnone, C., Korsic, M., et al. (2006). A systematic review of brain injury epidemiology in Europe. Acta neurochirurgica (Wien), 148, 255-268.

Thornhill, S., Teasdale, G. M., Murray, G. D., et al. (2000). Disability in young people and adults 1 year after head injury: prospective cohort study. BMJ, 320, 1631-1635.

Wallace, D. M., Shafazand, S., Ramos, A. R., Carvalho, D. Z., Gardener, H., Lorenzo, D., Wohlgemuth, W. K. (2011). Insomnia characteristics and clinical correlates in Operation Enduring Freedom/Operation Iraqi Freedom veterans with post-traumatic stress disorder and mild traumatic brain injury: An exploratory study. Sleep Med (Epub ahead of print).

Wilde, M. C., Castriotta, R. J., Lai, J. M., Atanasov, S., Masel, B. E., \& Kuna, S. T. (2007). Cognitive impairment in patients with traumatic brain injury and obstructive sleep apnea. Archives of Physical Medicine and Rehabilitation, 88, 1284-1288. 